Planetary Systems in the Universe - Observation, Formation and Evolution

Proceedings IAU Symposium No. 202, (C2004 IAU

Alan Penny, Pawel Artymowicz, Anne-Marie Lagrange, \& Sara Russell, eds.

\title{
The DARWIN project - An ESA cornerstone candidate mission
}

\section{C.V.M. Fridlund}

Space Science Department of the European Space Agency, ESTEC, PO Box 299, NL-2200AG, Noordwijk, The Netherlands

\begin{abstract}
The DARWIN project is ESA's candidate mission for the search for extra-solar Earth-type planets. Its aims are to survey the closest stars for these planets, and if detected characterise them as what concerns their physical conditions, their atmospheric composition and their ability to host life as we know it.
\end{abstract}

\section{Introduction}

The Infra Red Space Interferometer DARWIN was studied, by the European Space Agency (ESA), at system level, between 1997 and 2000. The study, carried out by Alcatel space division in Cannes, France has focused on developing a system that could carry out two main scientific objectives:

1. The detection and characterisation of Earth-like planets orbiting other stars

2. The imaging of astrophysical objects with unprecedented spatial resolution.

The most challenging of these objectives consist of the recording of infrared spectra of terrestrial exo-planets that could detect signs of biological activity at distances up to 20pc. In order to do this, the DARWIN project is constructed around the new technique of nulling interferometry, which exploits the wave nature of light to extinguish the light from a bright object (the central star, the primary, in this case). At the same time the light from a nearby source (the planet) is enhanced. The contrast between planets and stars being the least in the infrared wavelength region, that has been chosen for this mission. The result of the study was presented to the community in September of 2000 in Paris, France. A conference discussing the scientific issues was also held in Stockholm in November 1999.

\section{Formulation of the problem}

The main problem involved in the direct detection of an exo-planet of a size comparable with our own Earth and located at a similar distance from its own star is mainly one involving contrast \& dynamical range. The central star (the Sun, the primary) outshines the planet in the visual wavelength range by a factor of at least $10^{9}$. This problem is alleviated by going to the mid-infrared 
where the planets thermal emission peaks (a terrestrial planet is in this case defined as one of roughly the same size as the Earth and of the same surface temperature, $\approx 270 \mathrm{~K}$, thus having its peak emission at $10 \mu \mathrm{m}$. Even at these wavelengths, the contrast is more than a factor of $10^{6}$. The star and planet will be very near each other on the sky, and we need to devise a way of extinguishing the light from the star. Different coronographic methods have been evaluated, and albeit having the capability of achieving the scientific objective of detecting the exoplanet in some cases, these methods do not lend themselves to a large enough search space (unless the telescope is extremely large). Two other methods that have been suggested are 'nulling interferometry' and 'densified pupil' techniques. Of these, the 'nulling interferometry' technique was selected for the DARWIN study because of its relative simplicity and maturity (at the time).

\section{Nulling Interferometry}

Nulling interferometry can simply be described by considering two apertures, separated by a baseline $\mathrm{D}$. One now point the two telescopes towards the same star, and connects the light output of the two apertures. If one then make the optical path lengths of both apertures the same, the amplitudes of the electromagnetic radiation will interfere. This is interferometry in the classical sense producing a set of dark and bright bands - so called fringes. If we instead now make the light from one of the telescopes arrive at the site of beam combination with an added phase shift, $\pi$, the light along the optical axis will instead interfere destructively (the dark fringe appear 'on top of the star'). At the same time, waves arriving from a small angle, $\theta$, (which will depend on the separation between the two telescopes) away will interfere constructively. If we now assume that we have a star, which is orbited by a planet located at an angle, $\theta$, we can extinguish the light from the primary and isolate the stellar light. The contrast between the star and planet is now constrained to the light leaks from the 'central null caused by imperfections in optics and jitter of mechanical components. By using more telescopes it is possible to create a more complex transmission pattern. The actual pattern depends on the number of apertures, and the geometrical configuration. In the DARWIN configuration with 6 telescopes in a hexagonal pattern, with all telescopes equidistant from a central beam combiner, the pattern is roughly circular, with varying transmission along the parameter. It is now possible to 'tune the array to each individual star that is observed, so that the transmission ring is located in the so called habitable zone (see below). The signal also need to be modulated, in order to separate out an eventual planetary signal from any background (such as exo-zodiacal light) or to discriminate between different combinations of planets in the observed system. This can be performed either by rotation of the array of telescopes, switching between different combinations of apertures or a combination of both (the latter is the method foreseen to be used by DARWIN). 


\section{Search for extra-terrestrial life by remote sensing}

A major goal of the mission is not only to detect terrestrial exoplanets, but also to investigate if the conditions on the planet in question would allow life as we know it to exist and indeed if it already exist. In order to do so we need to define what life is, and how life as we understand it interact with its environment in an observable way. Life contains information; life is self-replicating; life evolves; and life affects its environment. We are attempting to detect life through remote sensing, at stellar distances. At first this goal seems hopeless. It has been found, however, that the simultaneous detection of water $\mathrm{H}_{2} \mathrm{O}$ oxygen and at a temperature of about $300 \mathrm{~K}$ is a clear indication of life as we know it. This is because oxygen is one of the most reactive substances there is. If all life on the Earth was removed suddenly (bacteria, green plants etc) all of the free oxygen in the Earth's atmosphere would disappear in the geologically short time of 4 million years. The criterion on temperature will define a so called habitable zone around each individual star. Strictly speaking, the surface temperature of a planet will depend not only on the energy input from the primary, but also on the atmospheric pressure and composition. Our own Earth, for instance, would be significantly colder without its green house effect, caused by e.g. $\mathrm{CO}_{2}$ and $\mathrm{CH}_{4}$. Since we a priori have no idea about the presence and/or composition of any eventual atmospheres, we will have to use this criterion with some care in the individual case.

We mentioned above that we wish to observe in the mid-IR in order to make the detection problem more tractable because of a lessened contrast. Within the wavelength range of $5 \mu$ and $20 \mu$ we find important absorption features of water, carbon dioxide and ozone. Ozone has been shown to be a good tracer of oxygen since its absorption feature in the spectrum show a logarithmic dependence on the abundance of molecular oxygen. With a spectral resolution, $\Delta \lambda / \lambda=20-$ 40 , we can detect all of the above mentioned features. We have to remember, that this refers to our Earth as it is today. The oxygen level in our atmosphere have been relatively high only for the last $20 \%-30 \%$ of the Earths history. Although, it is now more or less generally accepted that life arose on the Earth immediately after the era of bombardment, i.e. $3.8 \times 10^{9}$ years ago, it remained in the sea, and at a relatively simple level until just about 600 million years ago. This was the time of the so-called Cambrian explosion, when the phyla we can see today had their roots. Life until then was dominated by methane producing species and if we want to define our 'remote sensing criterion such that life is indicated by a disturbing of the equilibrium of a terrestrial planets atmosphere, we need to also take this evolutionary aspect into account.

\section{The DARWIN mission}

The current mission scenario is to be taken as a model that can fulfil the stated scientific objectives, but which does not include aspects related to international collaboration with e.g. NASA. It consist of six $1.5 \mathrm{~m}$ telescopes, which transmit their input beams to a beam combiner satellite. The individual telescopes each an individual satellite are kept to within one $\mathrm{cm}$ of their relative positions by utilising a metrology system, which includes laser metrology, radio frequency 
goniometry, and the tracking of interferometric fringes from a bright guide star. In the case of the planet finding part of the mission this is not going to be a problem, since the search for exo-planets will be carried out around relatively nearby and thus relatively bright stars. Adjustment of path lengths and the required phase delay is then introduced in the beam combiner satellite, where detection is also carried out. The system operates between wavelengths of $>5 \mu$ and $<20 \mu$, and thus require passive cooling of all optical components to below $40 \mathrm{~K}$. Because of this requirement, all power generating functions (computing, transmitting of data to Earth) is carried out on an non-cooled communications satellite. All 8 space craft are deployed and kept flying in formation in an orbit around the L2 Sun-Earth Lagrangian point. Launch is foreseen with an Ariane 5, and the DARWIN parameters are well within the envelope of that launcher. In the planet finding mode, DARWIN utilises baselines between $40 \mathrm{~m}$ and $250 \mathrm{~m}$.

A separate beam combiner table on the central space craft is used for 'normal' Michelson interferometry in an imaging mode. In imaging mode, baselines up to $1 \mathrm{~km}$ are foreseen. In the imaging mode, objects such as star forming regions, Active Galactic Nuclei, the core of our own Galaxy, Black Holes and very early galaxy formation, will be studied at unprecedented spatial resolution.

An ambitious technology program has been initiated in order to develop required items such as the nulling interferometry, metrology, and formation flying. Development of the required optical components such as polarisers, beam splitters and achromatic phase shifters are also being initiated. A test flight of selected technology is foreseen on the SMART-2 precursor mission in 2006. Collaboration with the European Southern Observatory (ESO) to validate nulling from the ground, and to carry out required precursor observations, is also being started.

Scientific collaboration and discussions have also been initiated with NASA's Terrestrial Planet Finder (TPF) with the ultimate goal of attempting a joint DARWIN-TPF mission. 